\title{
The Multi-slice Spiral Computed Tomography (MSCT) Features of Thin-Walled Cystic Lung Cancer with Ground Glass Opacity
}

\author{
Yejun $\mathrm{Yu}^{1,}{ }^{*}$ \\ ${ }^{1}$ Department of Radiology, 1st People's Hospital of Fuyang District of Hangzhou City Zhejiang Province, China \\ "Corresponding author: 1st People's Hospital of Fuyang District of Hangzhou City Zhejiang Province, 311400, China. Email: d73khh@163.com
}

Received 2020 May 02; Revised 2021 April 14; Accepted 2021 April 28.

\begin{abstract}
Background: Lung cancer is the leading cause of cancer-related mortality worldwide. Thin-walled cystic lung cancer with ground glas s opacity (GGO) is a type of lung carcinoma with specific computed tomography (CT) features.

Objectives: To investigate the imaging features of multi-slice spiral computed tomography (MSCT) of GGO and to improve the imaging diagnosis of this type of lung cancer.

Patients and Methods: The clinical data of 24 patients with pathologically confirmed thin-walled cystic lung cancer with GGO were retrospectively analyzed in this study. The imaging features of preoperative MSCT, including vascular convergence, burr signs, pleural depression signs, lobulation signs, thickness and uniformity of the cavity wall, partitive membranes, and position of the ground-glass cavity, were examined. The relationship between the imaging features and postoperative pathological findings of lung carcinoma were also explored.

Results: Most of the lesions (75.0\%) were located in the upper lobe of the lungs. The size range of the cavity was $5.8-28.1 \mathrm{~mm}$, with a mean of $13.6 \pm 7.2 \mathrm{~mm}$. Fifteen patients (62.5\%) had a cavity wall thickness $<2 \mathrm{~mm}, 6(25.0 \%)$ patients had a cavity wall thickness in the range of $2-3 \mathrm{~mm}$, and $3(12.5 \%)$ patients had a cavity wall thickness of about $3-4 \mathrm{~mm}$. A uniform cavity wall was found in 10 (41.7\%) cases, while a non-uniform cavity wall was observed in 14 (58.3\%) cases. Eleven patients showed pure GGO (45.8\%), while 13 patients showed mixed GGO (54.2\%). Besides, nine patients (37.5\%) showed lobular signs, 12 (50.0\%) patients showed spicule signs, and $6(28.6 \%)$ patients showed vascular convergence signs. Twelve patients (50.0\%) had signs of pleural indentation, while 14 (66.7\%) patients showed thick or thin small blood vessel opacity or fine partitive membranes. Twenty-three patients were diagnosed with lung adenocarcinoma, while one patients was diagnosed with mixed adenosquamous carcinoma.

Conclusion: Thin-walled cystic lung cancer with GGO is a rare clinical phenomenon. The imaging features of this type of lung cancer on MSCT are consistent with the characteristics of lung cancers.
\end{abstract}

Keywords: Lung Carcinoma, MSCT, Thin-Walled Cystic Lung Cancer, Ground Glass Opacity

\section{Background}

According to the data published by China Cancer Registration Center in 2014, 605,900 new cases of lung cancer (416,300 males and 189,600 females) were reported in China in 2010. According to recent lung cancer epidemiology findings, lung carcinoma is the leading malignancy in men and the third most common malignancy in women, accounting for $22.5 \%$ of all new cases of malignant tumors worldwide (31.5\% in men and $14.6 \%$ in women) (1).

Thin-walled cystic lung cancer is a type of lung carcinoma with special computed tomography (CT) features (2, 3 ). This type of lung cancer is defined as an air-containing space, surrounded by a thin wall ( $\leq 4 \mathrm{~mm}$ ) (3). However, the formation mechanism of thin-walled cystic lung cancer remains unclear (4). The majority of previous studies have reported that the development of thin-walled cystic lung cancer involves infiltration of the lung adenocarcinoma tissue into the alveolar wall and fine bronchial tubes to thicken the alveolar wall or protrude into the lumen, resulting in small airway stenosis and/or check-valve airway obstruction $(5,6)$. The breathing process continues to increase the internal gas volume and pressure to form a cyst cavity that continues to enlarge outside the tumor with lower pressure. However, this type of lung cancer is rarely seen in clinical practice (7).

\section{Objectives}

Due to its uncommon occurrence, thin-walled cystic lung cancer can be easily misdiagnosed. Therefore, in this 
study, we aimed to retrospectively analyze 24 cases of thinwalled cystic lung cancer, receiving surgical treatment in our hospital and to explore the imaging features of these patients.

\section{Patients and Methods}

\subsection{Patient Selection}

We retrospectively analyzed 24 cases of pathologically confirmed thin-walled cystic lung cancer with ground glass opacity (GGO) from September 2014 to February 2017 in our hospital. The inclusion criteria were as follows: (1) CT scan indicating a thin-walled translucent area, located at the center or on one side of GGO; (2) an average cavity wall thickness of $\leq 4 \mathrm{~mm}$; (3) treatment by surgery and pathologically confirmed diagnosis of lung cancer postoperatively; and (4) complete clinical and imaging data. The exclusion criteria were as follows: (1) cavity wall thickness > $4 \mathrm{~mm}$; (2) pulmonary bulla, pulmonary cyst, or thin-walled cystic lung cancer without GGO; (3) lack of a pathological diagnosis; and (4) incomplete clinical or imaging data.

\subsection{Multi-slice Spiral Computed Tomography (MSCT) Examina- tion}

A Philips Brilliance 16-slice spiral CT scanner(Philips CT Imaging Systems, Amsterdam, Netherlands) and a Siemens 64-slice dual-source CT system (Siemens Medical Imaging System, Munich, Germany) were used in the present study. The patients were placed in a supine position; the scanning range was from the chest entrance to the costal angle. The thickness of the scanning layer was 5-8 mm, with an interval of $4-6 \mathrm{~mm}$. Scanning was followed by a 1.5- to 2-mm thin-layer reconstruction. Five patients underwent enhanced CT examinations.

A high-pressure syringe was used to inject the contrast agent (Iopamidol, Bracco Co., Ltd., Shanghai, China) through the cubital vein as a bolus. The injection dose was $1.5 \mathrm{~mL} / \mathrm{kg}$, the injection flow rate was $2.6-3.0 \mathrm{~mL} / \mathrm{s}$, and the biphasic scanning time windows were 25 - 30 and 65 - 80 sec. The scanned raw data were forwarded to a workstation for multiplanar reconstruction (MPR). Continuous thinlayer CT examination of the cavity wall was performed, and the thickest and the thinnest cyst walls were selected through visual inspection. The wall thickness was measured with the CT workstation software by calculating the average of the thinnest and thickest parts.

\subsection{Statistical Analysis}

Statistical analysis was performed in SPSS version 17.0 (SPSS Inc., Chicago, IL, USA). Numerical variables are expressed as mean \pm standard deviation (SD). Quantitative data are expressed as relative numbers.

\section{Results}

\subsection{Patients' Characteristics}

Of 24 patients included in this study, 18 were male, and six were female; the male-to-female ratio was 3:1. The patients' age ranged from 46 to 75 years, and their mean age was $60.0 \pm 7.0$ years. Fifteen patients were smokers, 16 showed a productive cough, two patients showed cough accompanied by hemoptysis, and six patients had no obvious symptoms (Table 1 ).

\begin{tabular}{|c|c|}
\hline Characteristics & No. (\%) \\
\hline \multicolumn{2}{|l|}{ Age, $y$} \\
\hline $40-50$ & $3(12.5)$ \\
\hline $50-60$ & $8(33.3)$ \\
\hline $60-70$ & $9(37.5)$ \\
\hline$>70$ & $4(16.7)$ \\
\hline \multicolumn{2}{|l|}{ Gender } \\
\hline Male & $18(75.0)$ \\
\hline Female & $6(25.0)$ \\
\hline \multicolumn{2}{|l|}{ Smoking } \\
\hline Yes & $15(62.5)$ \\
\hline No & $9(37.5)$ \\
\hline \multicolumn{2}{|l|}{ Symptoms } \\
\hline Cough and expectoration & $16(66.7)$ \\
\hline Hemoptysis & $2(8.3)$ \\
\hline Asymptomatic & $6(25.0)$ \\
\hline \multicolumn{2}{|l|}{ Laboratory examination } \\
\hline Elevated carcinoembryonic antigen (CEA) & $9(37.5)$ \\
\hline Elevated CYFRA 21-1 & $7(29.2)$ \\
\hline \multicolumn{2}{|l|}{ Pathology } \\
\hline Adenocarcinoma & $23(95.8)$ \\
\hline Adenosquamous carcinoma & $1(4.2)$ \\
\hline
\end{tabular}

\subsection{Characteristics of Cavity Lesions}

All patients had single lesions. Six cases had lesions in the left upper lobe of the lungs, two cases had lesions in the left lower lobe of the lungs, 12 cases had lesions in the right upper lobe of the lungs, and four cases had lesions in the right lower lobe of the lungs. Most of the lesions (75.0\%) were located in the upper lobe of the lungs. The size range of the cavity was $5.8-28.1 \mathrm{~mm}$, with a mean of $13.6 \pm 7.2$ $\mathrm{mm}$. The thickness of the cyst wall was $0.4-3.6 \mathrm{~mm}$, with a mean of $1.7 \pm 0.9 \mathrm{~mm}$. Most patients had irregular cavity shapes (round or round-like). Fifteen patients (62.5\%) had 
a cavity wall thickness $<2 \mathrm{~mm}, 6(25.0 \%)$ patients had a cavity wall thickness range of $2-3 \mathrm{~mm}$, and $3(12.5 \%)$ patients had a cavity wall thickness of about $3-4 \mathrm{~mm}$. A uniform cavity wall was observed in 10 (41.7\%) patients, while a nonuniform cavity wall was identified in 14 (58.3\%) patients (Table 2).

\begin{tabular}{|c|c|}
\hline MSCT features & No. (\%) \\
\hline \multicolumn{2}{|l|}{ Lesion location } \\
\hline Left upper lung & $6(25.0)$ \\
\hline Left lower lung & $2(8.3)$ \\
\hline Right upper lung & $12(50.0)$ \\
\hline Right lower lung & $4(16.7)$ \\
\hline \multicolumn{2}{|l|}{ Cavity size (mm) } \\
\hline Range & $5.8-28.1$ \\
\hline Mean & $13.6 \pm 7.2$ \\
\hline \multicolumn{2}{|l|}{ Thickness of the cyst wall (mm) } \\
\hline Range & $0.4-3.6$ \\
\hline Mean & $1.7 \pm 0.9$ \\
\hline \multicolumn{2}{|l|}{ Cavity wall } \\
\hline Uniform & $10(41.7)$ \\
\hline Non-uniform & $14(58.3)$ \\
\hline \multicolumn{2}{|l|}{ GGO type } \\
\hline Pure GGO & $11(45.8)$ \\
\hline Mixed GGO & $13(54.2)$ \\
\hline \multicolumn{2}{|l|}{ Signs of GGO } \\
\hline Lobular sign & $9(37.5)$ \\
\hline Spicule sign & $12(50)$ \\
\hline Vascular convergence & $6(25.0)$ \\
\hline Pleural indentation & $12(50.0)$ \\
\hline Small blood vessel opacity & $14(58.3)$ \\
\hline
\end{tabular}

Abbreviations: MSCT, multi-slice spiral computed tomography; GGO, ground glass opacity.

\subsection{Characteristics of GGO}

The maximum width range of GGO was 7.01-22.92 mm. Eleven patients showed pure GGO (45.8\%) (Figure 1A), while 13 patients showed mixed GGO (54.2\%) (Figure 1B). Nine patients (37.5\%) had lobular signs, 12 (50.0\%) patients showed spicule signs (Figure 2A), and 6 (28.6\%) patients showed vascular convergence signs (Figure $2 \mathrm{~B}$ ). Twelve patients (50.0\%) showed obvious signs of pleural indentation, while $14(58.3 \%)$ cases showed thick or thin small blood vessel opacity or fine partitive membranes (Table 2).

\subsection{Postoperative Pathology}

Twenty-three patients were pathologically diagnosed with lung adenocarcinoma (Figure 3A), while one patient was diagnosed with mixed adenosquamous carcinoma (Figure 3B).

\subsection{Follow-up}

The follow-up period ranged from 39 to 69 months postoperatively. Until June 2020, 16 patients expired, while eight patients were still alive.

\section{Discussion}

Pulmonary cystic lesions are defined as gas-containing translucent areas with a wall thickness of $\leq 4 \mathrm{~mm}$. On the other hand, a cavitary lung lesion is defined as a gascontaining translucent area with a thicker wall than cystic lung lesions $(>4 \mathrm{~mm})(8,9)$. Although cavities are commonly seen in lung cancer patients, accounting for $22 \%$ of all primary lung carcinomas (9), thin-walled cystic lung cancer has been rarely reported in the literature. Cavitary lung cancer is common in patients with squamous cell carcinoma (SCC) and is often seen in adenocarcinomas (10). Occasionally, it can be found in bronchioloalveolar carcinoma (BAC), while small-cell lung cancer has not been associated with cavitary lesions (10).

Today, formation of gas-bearing lacunae is believed to occur through several mechanisms: (1) tumor tissue invasion of the original lung cyst or bullae; (2) ischemic necrosis of tumor tissue and excretion of the liquefied part through the bronchus to form a cystic translucent area; and (3) one-way valve formation. According to the characteristics of patients in the present study, as well as previous publications, one-way valve formation is believed to be the main mechanism of cystic lung cancer development with peripheral GGO.

The evolution of GGO lesions from atypical adenomatous hyperplasia (AAH) to adenocarcinoma in situ (AIS) and to minimally invasive adenocarcinoma (MIA) is an evolving and progressive process. As it further infiltrates the lung tissue, tumor invades the alveolar wall, bronchiolar wall, and interstitial lung, forming a one-way valve that continuously accumulates gas in the alveolar space. Multiple alveolar spaces then burst and fuse into a cystic cavity; over time, pressure inside the cyst increases, and its volume expands.

The epidermal growth factor (EGFR) is believed to be involved in multiple processes, such as tumor infiltration, angiogenesis, and cell proliferation (11). A previous study reported that $81 \%$ of all thin-walled cystic lung cancers are associated with EGFR overexpression (12). In the present 


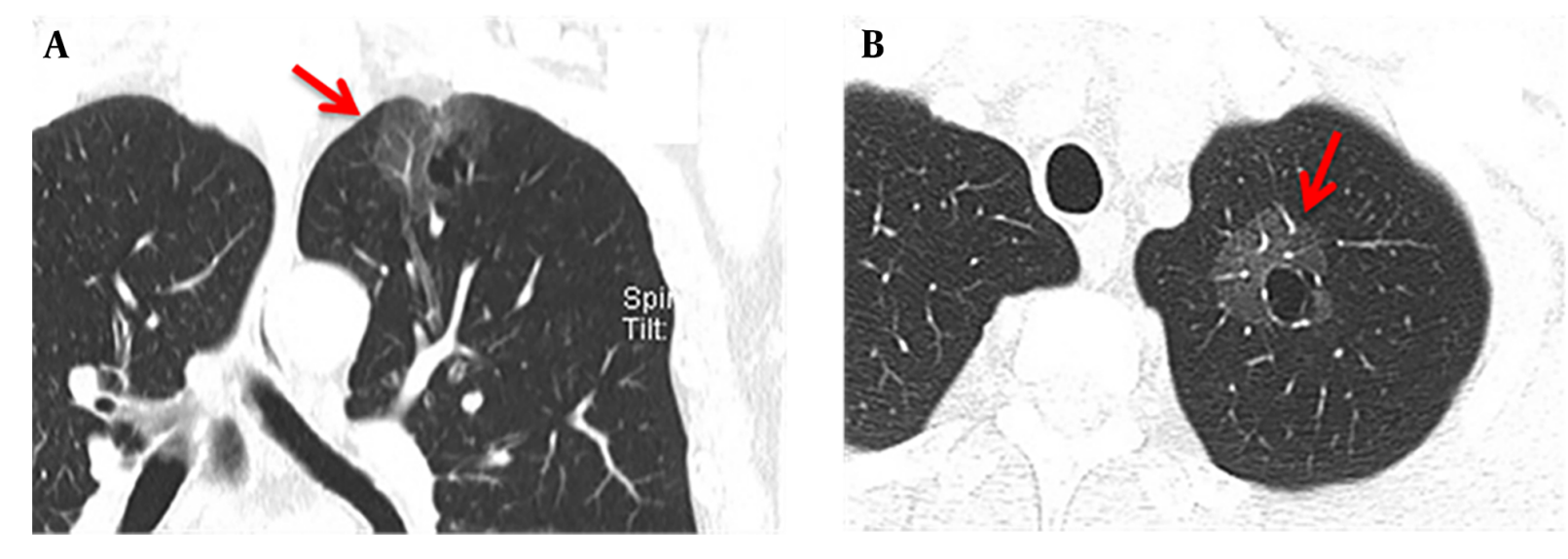

Figure 1. A 66-year-old man with more than one month of coughing. The multi-slice spiral computed tomography (MSCT) scan showed a round cavity in the upper lobe of the left lung with pure ground glass opacity (GGO). A: Coronal MSCT images showed diaphragmatic and pleural depressions, without an obvious burr sign (red arrow). B: A thin wall cavity was found in the left upper lobe of the lung with mixed GGO (red arrow). The CT scan showed that the inner wall of the cavity was smooth, and the wall thickness was uniform with multiple septal shadows.
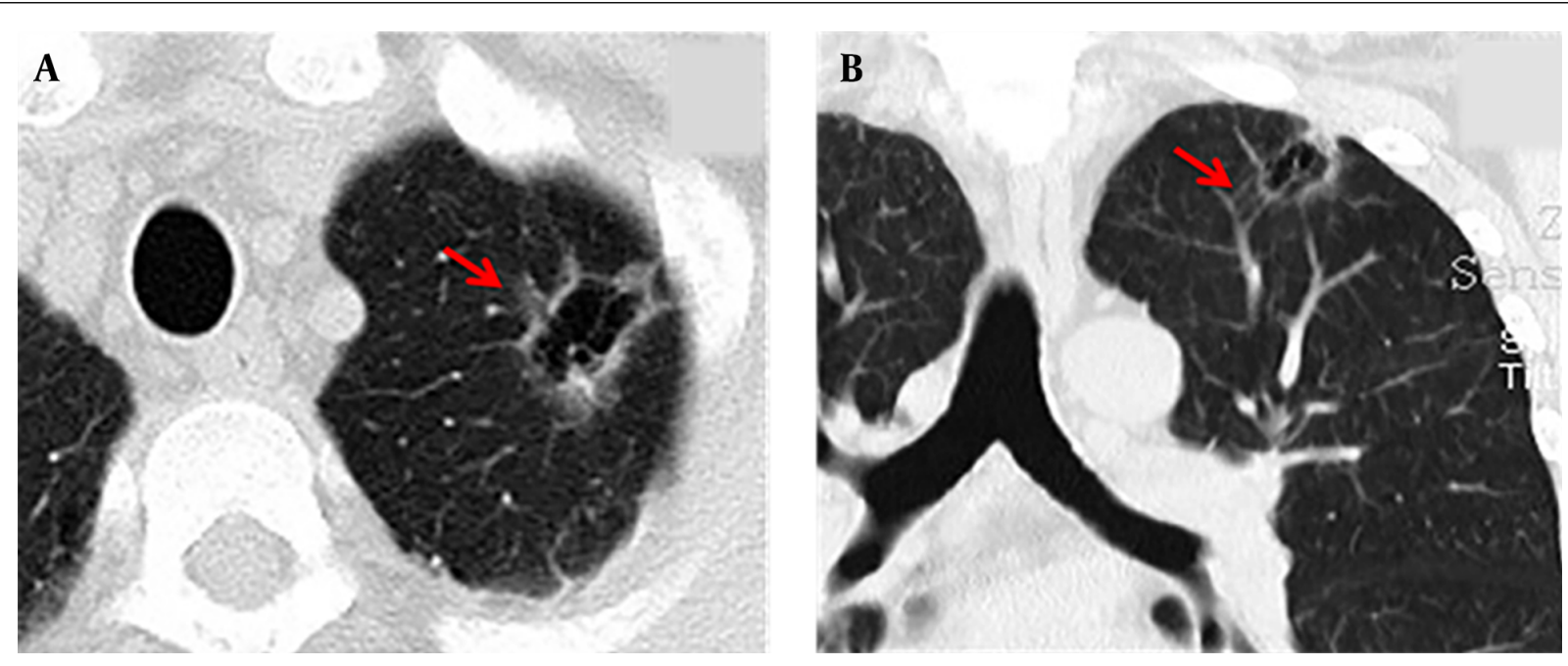

Figure 2. A 58-year-old man had symptoms of cough and expectoration for one week. The multi-slice spiral computed tomography (MSCT) scan showed an irregular cavity in the left upper lobe of the lung with spicule and vascular convergence signs. A: The CT scan showed that the lesion was located in the upper lobe of the left lung. The inner wall of the cavity was smooth, the thickness of the wall was not uniform, and internal septation was visible; the lesions were surrounded by ground glass opacity (GGO) and lobulated at the edge of the lesion (red arrow). B: The coronal view showed that the cavity was divided internally, blood vessels passed through the cavity, and the surrounding vessels were clustered (red arrow).

study, $18(75.0 \%)$ tumors were reported in the upper lobe of the lungs, as the preferred site for emphysema. Although tumor tissue invasion into the existing pulmonary cysts or large bulla structures has been proposed in the literature, this theory cannot be widely accepted, since lesions have not been found in the common sites of bullae, and this type of balloon-containing lumen has not been previously observed (13).

Some researchers believe that the soft and fragile nature of tumor tissue leads to the elastic retraction of the peripheral lung tissue and aggravates the valve mechanism
$(14,15)$. Microscopic analyses have indicated tumor infiltration along the walls of the bronchioles and cyst, but not the liquefied necrotic tissue. This observation better supports the mechanism of one-way valve formation in thin-walled cystic lung cancer, which is completely different from the mechanism of cavity formation in necrotic tumors, such as SCC.

In the present study, 14 out of 24 patients (58.3\%) showed fine partitions or small blood vessel shadows in the cavity, suggesting cavity formation as an expansionlike change, as cavitary lung cancer does not show par- 

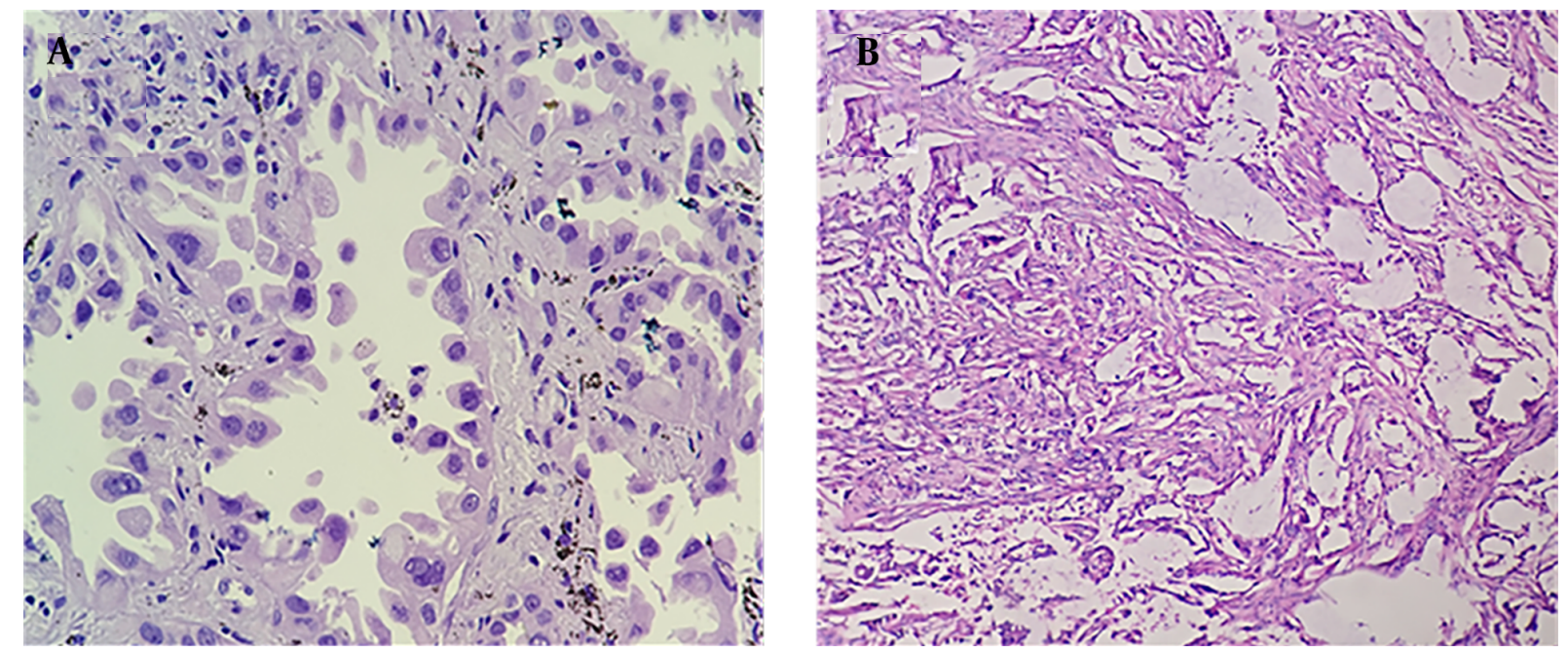

Figure 3. A: A 56-year-old woman received lobectomy for lung cancer treatment. Hematoxylin and Eosin (H\&E) staining showed lung adenocarcinoma, mainly distributed in the adenoid structure. Tumor cells grew along the alveolar wall in an adherent manner. The cell body was large, the nucleus was large and dark-stained, and the nucleus was irregular with nucleoli. B: A 60-year-old man with cough for one month undergoing surgery. Pathological examination demonstrated that the adenosquamous carcinoma tissue was distributed in nests, with a large cell body, an abundant cytoplasm, an eosinophilic, large, and deeply stained nucleus, and an irregular nuclear shape; in some areas, the tumor tissue had a glandular structure with heterotypic cells and infiltrative growth.

tition opacity due to complete liquefied necrosis. Since GGO lesions have the characteristics of malignancy, such as lobulation, vascular convergence, pleural depression, and burr signs, thin-layer CT scan and MPR can reflect these imaging features completely to indicate the exact location of the lesion, the size of the cavity, and the relationship of these lesions with peripheral tissues. Therefore, CT imaging is the preferred method for examining thin-walled cystic lung cancer with GGO.

Since it is difficult to distinguish between benign and malignant parenchymal lesions, fiberoptic bronchoscopy and percutaneous lung biopsy are suggested for pathological diagnosis. Considering the characteristics of thinwalled cystic lung cancer, its pathology may be challenging because of thin walls. Also, as inaccurate puncture can easily lead to pneumothorax. Some scholars have proposed the application of CT-guided percutaneous needle aspiration biopsy to avoid this complication (16).

Cavitary lung lesions may be associated with some diseases. They are commonly seen in SCC patients. The pathological mechanism of this type of cavitation involves the formation of liquefied tumor necrosis that creates a hollow area through bronchial discharge, forming a thickwalled cavity with an irregular internal surface. Wall nodules without internal partitions or vascular structures and lobulation signs may be also present. Besides, pleural depression, burr sings, and other malignant signs may be observed.

Moreover, pulmonary cysts or bullae are identified as thin-walled lung cysts. Internal partitions, lobulation signs, wall nodules, and other signs are absent in these cysts. During alveolar expansion, cystic cavity develops through rupture and fusion of the alveolar wall, usually caused by obstruction of a small bronchial valve. Besides, tuberculosis cavities mainly present as fibrous thickwalled cavities with extensive cord-like fibrotic changes around the lesions, scattered new and old lesions, calcification, pleural thickening, and other symptoms. Once $M y$ cobacterium tuberculosis is found in clinical sputum samples, identifying the disease is not difficult.

In this study, we reviewed the clinical and radiographic features of 24 patients with thin-walled peripheral GGO. The findings showed that most cases of lung cancer were adenocarcinomas. In terms of imaging features, $50 \%$ of the lesions showed pure GGO, while 50\% showed mixed GGO. Most of the lesions were associated with at least one sign of lobulation, burr sign, vascular convergence, or pleural depression. With a pathological understanding of the MSCT features of thin-walled cystic lung cancer with GGO, benign and malignant cases can be distinguished when encountering similar images (3).

Considering the prognosis of thin-walled cystic lung cancer, studies in the literature have reported small sample sizes with limited survival data. In our dataset until June 2020, 16 patients expired, while eight patients remained alive during the follow-up (39 - 69 months), with a three-year survival rate of 33.3\%. However, the survival rate was reported in a small sample size $(n=24)$, and further val- 
idation in a larger sample size is needed for survival studies. Therefore, similar cases should be examined, and prognosis needs to be further evaluated.

In conclusion, thin-walled cystic lung cancer with GGO is a rare clinical phenomenon. Adenocarcinoma is the main pathological diagnosis of this type of lung carcinoma. Although this cancer is usually a relatively lower grade malignancy with well or moderate tumor differentiation, early detection and surgical resection remain appropriate managment options.

\section{Footnotes}

Authors' Contribution: Yejun Yu designed and performed the study and prepared the manuscript.

\section{Clinical Trial Registration Code: NA.}

Conflict of Interests: None.

Ethical Approval: This study was approved by the Ethics Committee of the First People' s Hospital in Fuyang District of Hangzhou, Zhejiang Province, 311400, PR China.

Funding/Support: None.

Informed Consent: Informed consent was obtained from all participants.

\section{References}

1. International Agency for Research on Cancer. Global cancer observatory: Cancer today. Lyon, France: WHO,;2018, [cited 20 Aug 2020]. Available from: https://gco.iarc.fr/today.

2. Mergo PJ, Rojas CA. CT characteristics and pathologic basis of solitary cystic lung cancer. Radiology. 2019;291(2):502-3. doi: 10.1148/radiol.2019190329. [PubMed: 30860447].

3. Zhang J, Deng H, Wu CC, Wang Z, Zhao D, Wei B, et al. The mechanism of formation of thin-walled cystic lung cancer. Medicine (Baltimore). 2019;98(14). e15031. doi: 10.1097/MD.0000000000015031. [PubMed: 30946341]. [PubMed Central: PMC6455936].

4. Kolesov AP, Toluzakov VL, Krol Ia M, Izbinskii AL, Shteintsaig AI, Davydenko VA, et al. [On the differential diagnosis of cavernous cancer of the lung]. Vopr Onkol.1966;12(3):3-11. Russian. [PubMed: 5991960].
5. Palamarchuk VP, Krol Ia M, Davydenko VA, Bonk GM. [Radiography of cavernous forms of lung cancer]. Vestn Rentgenol Radiol.1968;43(2):1924. Russian. [PubMed: 5739373].

6. Deng H, Zhang J, Zhao S, Zhang J, Jiang H, Chen X, et al. Thin-wall cystic lung cancer: A study of 45 cases. Oncol Lett. 2018;16(1):75560. doi: 10.3892/ol.2018.8707. [PubMed: 29963142]. [PubMed Central: PMC6019975].

7. Woodring JH, Fried AM, Chuang VP. Solitary cavities of the lung: diagnostic implications of cavity wall thickness. AJR Am J Roentgenol. 1980;135(6):1269-71. doi: 10.2214/ajr.135.6.1269. [PubMed: 6779538].

8. Meyer L, Schuck A, Burgi U, Huber LC. [CME: Cavitary pulmonary disease - differential diagnosis and diagnostic work-up]. Praxis (Bern 1994). 2018;107(8):413-21. Ukrainian. doi: 10.1024/1661-8157/a002944. [PubMed: 29642791].

9. Gadkowski LB, Stout JE. Cavitary pulmonary disease. Clin Microbiol Rev. 2008;21(2):305-33. doi: 10.1128/CMR.00060-07. [PubMed: 18400799]. [PubMed Central: PMC2292573].

10. Opoka LM, Szturmowicz M, Oniszh K, Korzybski D, Podgajny Z, Blasinska-Przerwa K, et al. CT imaging features of thin-walled cavitary squamous cell lung cancer. Adv Respir Med. 2019;87(2):114-7. doi: 10.5603/ARM.2019.0018. [PubMed: 31038723].

11. Stella GM, Piloni D. Exploring adjuvant epidermal growth factor receptor inhibition in non-small cell lung cancer. Minerva Med. 2017;108(3 Suppl 1):6-12. doi: 10.23736/S0026-4806.17.05322-8. [PubMed: 28862415].

12. Onn A, Choe DH, Herbst RS, Correa AM, Munden RF, Truong MT, et al. Tumor cavitation in stage I non-small cell lung cancer: epidermal growth factor receptor expression and prediction of poor outcome. Radiology. 2005;237(1):342-7. doi: 10.1148/radiol.2371041650. [PubMed: 16183941].

13. Iwata T, Nishiyama N, Nagano K, Izumi N, Tsukioka T, Hanada S, et al. Squamous cell carcinoma presenting as a solitary growing cyst in lung: a diagnostic pitfall in daily clinical practice. Ann Thorac Cardiovasc Surg. 2009;15(3):174-7. [PubMed: 19597393].

14. Scholling S, Bauer T, Muller S, Troger I, Bartling A, Gillissen A, et al. [Differential diagnosis of cavernous lung structures]. Internist (Berl). 1997;38(9):858-61. German. doi: 10.1007/s001080050097. [PubMed: 9410834].

15. Lovrenski A, Panjkovic M, Eri Z, Klem I, Samardzija G, Milic M. [Cavernous hemangioma of the lung]. Med Pregl. 2011;64(5-6):327-30. Serbian. doi: 10.2298/mpns1106327l. [PubMed: 21789928].

16. Nakahara Y, Mochiduki Y, Miyamoto Y. Percutaneous needle washing for the diagnosis of pulmonary thin-walled cavitary lesions filled with air. Intern Med. 2007;46(14):1089-94. doi: 10.2169/internalmedicine.46.0020. [PubMed: 17634705]. 